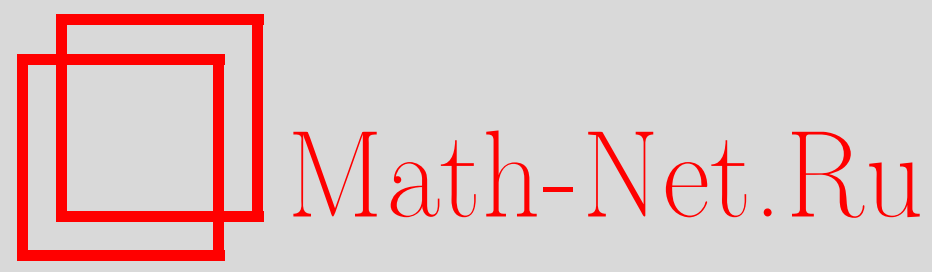

А. И. Галочкин, Оценки числа нулей некоторых функций с алгебраическими коэффициентами рядов Тейлора, $M a$ тем. заметки, 1997, том 61, выпуск 6, 817-824

DOI: https://doi.org/10.4213/mzm1566

Использование Общероссийского математического портала Math-Net.Ru подразумевает, что вы прочитали и согласны с пользовательским соглашением http://www . mathnet.ru/rus/agreement

Параметры загрузки:

IP: 3.80 .253 .173

26 апреля 2023 г., 18:02:18

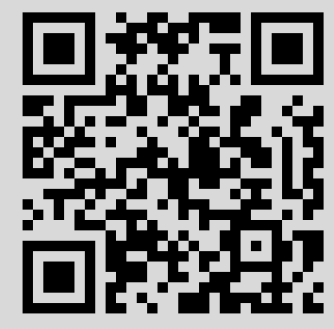




\section{ОЦЕНКИ ЧИСЛА НУЛЕЙ НЕКОТОРЫХ ФУНКЦИЙ С АЛГЕБРАИЧЕСКИМИ КОЭФФИЦИЕНТАМИ РЯДОВ ТЕЙЛОРА}

\section{А.И. Галочкин}

Доказываются две теоремы о числе нулей аналитических функций некоторых классов, в том числе $E$-функций и $G$-функций Зигеля. С помощью этих теорем дается новое доказательство теоремы Гельфонда-Шнейдера и улучшается результат о необращении в нуль числового определителя при доказательстве теоремы Шидловского.

Библиограффия: 5 названий.

Пусть $\mathbb{K}$ - алгебраическое числовое поле степени $\varkappa, \mathbb{Z}_{\mathbb{K}}$-кольцо целых чисел в поле $\mathbb{K}$, $\operatorname{Norm}(\alpha)$ - норма числа $\alpha \in \mathbb{K}$. Для аналитической функции $f(z)$ через $\operatorname{ord}_{z=\alpha} f(z)$ будем обозначать кратность ее нуля в точке $z=\alpha$.

Пусть функция

$$
f(z)=\sum_{\nu=0}^{\infty} a_{\nu} \frac{z^{\nu}}{\nu !}
$$

удовлетворяет следующим условиям:

1) все коэффициенты $a_{\nu} \in \mathbb{K}$;

2) для них и для сопряженньх им чисел в поле $\mathbb{K}$

$$
a_{\nu}^{[k]}, \quad k=\overline{1, \varkappa}, \quad a_{\nu}^{[1]}=a_{\nu}
$$

справедливы оценки

$$
\left|a_{\nu}\right|<\Lambda_{1} C_{1}^{\nu}, \quad \prod_{k=2}^{\varkappa}\left|a_{\nu}^{[k]}\right|<\Lambda_{2} C_{2}^{\nu}, \quad \nu=0,1, \ldots
$$

3) существует последовательность $\left\{q_{n}\right\}$ ненулевых цельх чисел в поле $\mathbb{K}$ такая, что

$$
q_{\nu} a_{\nu} \in \mathbb{Z}_{\mathbb{K}}
$$

и

$$
\left|\operatorname{Norm}\left(q_{\nu}\right)\right|<\Lambda_{3} C_{3}^{\nu}, \quad \nu=0,1, \ldots,
$$

где $\Lambda_{1}, \Lambda_{2}, \Lambda_{3}, C_{1}, C_{2}, C_{3}$ - положительные числа.

Работа выполнена при частичной поддержке Российского фонда фундаментальных исследований, грант № 94-01-00739. 
ТЕОРемА 1. Пусть функиия $f(z)$ удовлетворяет всем указанным выше условиям. Далее, пусть $N(R)$ - количество ее нулей в круге $|z| \leqslant R$ с учетом их кратности, в частности, $N(0)=\operatorname{ord}_{z=0} f(z)$. Тогда при любом положительном значении $R$ справедливо неравенство

$$
(N(R)-N(0)) \ln \left(2+\frac{N(0)}{C_{1} R}\right) \leqslant N(0)\left(1+\ln \left(C_{1} C_{2} C_{3}\right)\right)+3 C_{1} R+\ln \left(\Lambda_{1} \Lambda_{2} \Lambda_{3}\right)
$$

Теорема 1 применима, в частности, к E-функциям Зигеля, если их рассматривать в смысле определения, данного в $[1$, гл. $13, \S 1]$.

Подобная теорема справедлива также для другого класса функций, содержащего $G$-функции Зигеля (см. заключительные замечания в конце книги [1], а также классическую статью К. Зигеля [2]).

Пусть задана функция

$$
g(z)=\sum_{\nu=0}^{\infty} a_{\nu} z^{\nu}
$$

коэффициенты которой $a_{\nu}$ удовлетворяют тем же условиям, что и коэффициенты функции (1).

Формально $g(z)$ отличается от $f(z)$ отсутствием величины $\nu$ !. Однако $g(z)$, в отличие от $f(z)$, не является целой функцией.

ТЕОРема 2. Пусть функиия $g(z)$ удовлетворяет указанным выше условиям, $N(R)$ - количество ее нулей в круге $|z| \leqslant R$ с учетом их кратности, в частности, $N(0)=\operatorname{ord}_{z=0} g(z)$. Тогда при любом $R$ таком, что $0<R<1 /\left(3 C_{1}\right)$, выполняется неравенство

$$
(N(R)-N(0)) \ln \frac{1}{3 C_{1} R} \leqslant N(0) \ln \left(1.5 C_{1} C_{2} C_{3}\right)+\ln \left(3 \Lambda_{1} \Lambda_{2} \Lambda_{3}\right) .
$$

ДоКАЗАТЕЛЬСТВо ТЕОРЕМЫ 1 . Пусть $p=N(0)$, тогда в равенстве (1)

$$
f(z)=\sum_{\nu=p}^{\infty} a_{\nu} \frac{z^{\nu}}{\nu !}, \quad a_{p} \neq 0
$$

а значит,

$$
\left|\operatorname{Norm}\left(q_{p} a_{p}\right)\right| \geqslant 1
$$

где $q_{n}$ - числа, определенные с помощью (3). Следовательно,

$$
\left|a_{p}\right| \geqslant\left|\operatorname{Norm}\left(q_{p}\right) a_{p}^{[2]} \cdots a_{p}^{[\varkappa]}\right|^{-1} \geqslant\left(\Lambda_{2} \Lambda_{3}\right)^{-1}\left(C_{2} C_{3}\right)^{-p}
$$

Пусть теперь $\alpha_{1}, \ldots, \alpha_{n}$ - все другие нули функции $f(z)$, расположенные в круге $|z| \leqslant R$, т.е.

$$
\left|\alpha_{j}\right| \leqslant R, \quad f\left(\alpha_{j}\right)=0, \quad \alpha_{j} \neq 0, \quad j=\overline{1, n}, \quad n=N(R)-N(0) .
$$


Тогда в силу (7) при любых положительных числах $r$ и $\lambda$ справедливы равенства

$$
\begin{gathered}
a_{p}=f^{(p)}(0)=\frac{p !}{2 \pi i} \oint_{|z|=r} \frac{f(z)}{z^{p+1}} d z \\
f(z)=\frac{1}{2 \pi i} \oint_{|u|=\lambda p+3 R}\left(\left(\frac{z}{u}\right)^{p} \prod_{j=1}^{n}\left(\frac{z-\alpha_{j}}{u-\alpha_{j}}\right)\right) \frac{f(u) d u}{u-z} .
\end{gathered}
$$

Из (1) и (2) получаем, что

$$
\begin{gathered}
|f(u)| \leqslant \sum_{\nu=0}^{\infty} \Lambda_{1} C_{1}^{\nu} \frac{|u|^{\nu}}{\nu !} \leqslant \Lambda_{1} e^{C_{1}(\lambda p+3 R)} \quad \text { при } \quad|u|=\lambda p+3 R \\
\prod_{j=1}^{n}\left|\frac{z-\alpha_{j}}{u-\alpha_{j}}\right| \leqslant\left(\frac{R+r}{\lambda p+2 R}\right)^{n}
\end{gathered}
$$

поэтому из (9) следует, что

$$
\left|a_{p}\right| \leqslant \frac{p !}{(2 \pi)^{2}} 2 \pi r \cdot \frac{1}{r} 2 \pi(\lambda p+3 R)^{1-p}\left(\frac{R+r}{\lambda p+2 R}\right)^{n} \Lambda_{1} e^{C_{1}(\lambda p+3 R)}(\lambda p+3 R-r)^{-1} .
$$

Отсюда ввиду произвольности числа $r>0$ имеем

$$
\left|a_{p}\right| \leqslant \Lambda_{1} \lambda^{-p}\left(2+\frac{\lambda p}{R}\right)^{-n} e^{C_{1}(\lambda p+3 R)}
$$

что вместе с неравенством (8) и равенствами $p=N(0), n=N(R)-N(0)$ приводит к оценке

$$
\begin{aligned}
& (N(R)-N(0)) \ln \left(2+\frac{\lambda N(0)}{R}\right) \\
& \quad \leqslant N(0)\left(C_{1} \lambda-\ln \lambda+\ln \left(C_{2} C_{3}\right)\right)+3 C_{1} R+\ln \left(\Lambda_{1} \Lambda_{2} \Lambda_{3}\right) .
\end{aligned}
$$

Полагая в (10) $\lambda=C_{1}^{-1}$, получаем неравенство (5). Теорема доказана.

Приведенные здесь и в следующем далее доказательстве теоремы 2 рассуждения близки к доказательству теоремы Иенсена (см., например, [3, гл. 1, § 1.1]).

ДоКАЗАТЕЛЬСТВО ТЕОРЕМЫ 2 близко к доказательству теоремы 1 , поэтому проведем его менее подробно.

Как и ранее, справедливо неравенство (8). Соотношения (9) заменяются на равенства

$$
\begin{gathered}
a_{p}=\frac{g^{(p)}(0)}{p !}=\frac{1}{2 \pi i} \oint_{|z|=r} \frac{g(z)}{z^{p+1}} d z \\
g(z)=\frac{1}{2 \pi i} \oint_{|u|=c}\left(\left(\frac{z}{u}\right)^{p} \prod_{j=1}^{n}\left(\frac{z-\alpha_{j}}{u-\alpha_{j}}\right)\right) \frac{g(u) d u}{u-z}
\end{gathered}
$$


где $c=\left(1.5 C_{1}\right)^{-1}, 0<r<c$, а числа $p, n, \alpha_{1}, \ldots, \alpha_{n}$ имеют тот же смысл, что и в теореме 1 . На этот раз в силу (2) $|g(u)| \leqslant 3 \Lambda_{1}$,

$$
\left|\prod_{j=1}^{n}\left(\frac{z-\alpha_{j}}{u-\alpha_{j}}\right)\right| \leqslant\left(\frac{R+r}{c-R}\right)^{n}
$$

Поэтому

$$
\left|a_{p}\right| \leqslant \frac{1}{(2 \pi)^{2}} 2 \pi r \cdot \frac{1}{r} 2 \pi c^{1-p}\left(\frac{R+r}{c-R}\right)^{n} \frac{3 \Lambda_{1}}{c-r}
$$

что при $r \rightarrow+0$ приводит к неравенству

$$
\left|a_{p}\right| \leqslant 3 \Lambda_{1} c^{-p}\left(\frac{R}{c-R}\right)^{n}
$$

и с помощью (6) и соотношений $c=\left(1.5 C_{1}\right)^{-1}$ и $0<R<\left(3 C_{1}\right)^{-1}$ позволяет завершить доказательство теоремы.

Теорема 1 дает возможность предложить новое доказательство знаменитой теоремы Гельфонда-Шнейдера, давшей решение седьмой проблемы Гильберта. В отличие от других доказательств, на первом этапе строится многочлен, имеющий "большой" порядок нуля только в точке $z=0$, а не на некотором множестве точек. После этого оценка (5) быстро приводит к противоречию. Процесс интерполяции не применяется.

ТЕОРЕМА ГЕЛЬФОНДА-ШНЕЙДЕРА. Пусть $а$ u $\alpha-$ алгебраические числа, $\alpha \notin \mathbb{Q}$ и $\alpha \ln a \neq 0$. Тогда число $a^{\alpha}$ трансиендентно.

ЛЕмма 1. Система уравнений

$$
\begin{gathered}
L_{i}=\sum_{j=1}^{q} a_{i j} x_{j}=0, \quad i=\overline{1, p}, \\
a_{i j} \in \mathbb{Z}_{\mathbb{K}}, \quad \mid a_{i j} \leqslant A, \quad p<q,
\end{gathered}
$$

имеет ненулевое решение $\left(x_{1}, \ldots, x_{q}\right)$ в $\mathbb{Z}_{\mathbb{K}}$, удовлетворяющее неравенству

$$
\left|x_{j}\right|<C(C q A)^{p /(q-p)}, \quad j=\overline{1, q},
$$

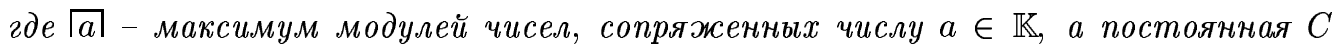
зависит только от поля $\mathbb{K}$.

Доказательство этой известной леммы Зигеля можно найти, например, в [1, гл. $3, \S 8]$.

Через $\gamma_{1}, \gamma_{2}, \ldots$ будем обозначать положительные постоянные, не зависящие от параметра $n$. 
ЛЕмма 2. Если число $\alpha$ алгебраическое $и \mathbb{K}=\mathbb{Q}(\alpha)$, то существует функиия

$$
F(z)=\sum_{k=0}^{n-1} \sum_{l=0}^{n-1} A_{k l} e^{(k+l \alpha) z}
$$

такая, что все числа $A_{k l} \in \mathbb{Z}_{\mathbb{K}}$

$$
0<\max \left|A_{k l}\right|<e^{\gamma_{1} n^{2} / \ln ^{3} n}
$$

$u$

$$
F^{(\nu)}(0)=0, \quad \nu=\overline{0, p-1}, \quad p=\left[\frac{n^{2}}{\ln ^{2} n}\right] .
$$

ДОКАЗАТЕЛЬСТВО леммы состоит в применении леммы 1 к системе (13), состоящей из $p=\left[n^{2} / \ln ^{2} n\right]$ уравнений относительно $q=n^{2}$ неизвестных $A_{k l}$.

Лемма 3. Пусть $а, \alpha$ и $a^{\alpha}$ - алгебраические числа, $\mathbb{K}=\mathbb{Q}\left(a, \alpha, a^{\alpha}\right), \varkappa=\operatorname{deg} \mathbb{K}$ и $F(z) \not \equiv 0$ - функция вида (11), для коэффичиентов $A_{k l}$ которой выполняются неравенства (12). Пусть $S$ - фиксированное натуральное число, $p_{s}$ - порядок нуля функиии $F(z)$ в точке $s \ln a, s=\overline{0, S}$. Тогда для любого числа $\varepsilon>0$ и любого индекса $s_{0}, 0 \leqslant s_{0} \leqslant S$, при $n>n_{0}(\varepsilon)$ выполняется неравенство

$$
P\left(s_{0}\right)=\sum_{\substack{s=0 \\ s \neq s_{0}}}^{S} p_{s} \leqslant(\varkappa+\varepsilon) p_{s_{0}}+\gamma_{2} \frac{n^{2}}{\ln ^{3} n} .
$$

ДокАЗАТЕЛЬСТВо. Полагая $u=z-s_{0} \ln a$, получаем разложение

$$
F(z)=\sum_{\nu=0}^{\infty} \frac{F^{(\nu)}\left(s_{0} \ln a\right)}{\nu !} u^{\nu}
$$

функции $F(z)=F\left(u+s_{0} \ln a\right)=f(u)$ в ряд по степеням $u$. К функции $f(u)$ применим теорему 1. В этой теореме

$$
\begin{gathered}
R=S|\ln a|=\text { const, } \quad N(0)=p_{s_{0}}, \\
a_{\nu}=F^{(\nu)}\left(s_{0} \ln a\right), \quad N(R)-N(0) \geqslant P\left(s_{0}\right) .
\end{gathered}
$$

Далее,

$$
f^{(\nu)}(0)=\sum_{k=0}^{n-1} \sum_{l=0}^{n-1} A_{k l}(k+l \alpha)^{\nu} e^{(k+l \alpha) s_{0} \ln a} .
$$

Поэтому в теореме 1 в силу (12)

$$
\begin{gathered}
|k+l \alpha| \leqslant \gamma_{3} n=C_{1}=C_{2}^{1 /(\varkappa-1)}, \\
\left|A_{k l} e^{(k+l \alpha) s_{0} \ln a}\right| \leqslant e^{\gamma_{4} n^{2} / \ln ^{3} n}=\Lambda_{1}=\Lambda_{2}^{1 /(\varkappa-1)} .
\end{gathered}
$$


Наконец, если $b$ - такое натуральное число, что $b a, b \alpha, b a^{\alpha} \in \mathbb{Z}_{\mathbb{K}}$, то в (16)

$$
b^{2 n S+\nu} F^{(\nu)}\left(s_{0} \ln a\right) \in \mathbb{Z}_{\mathbb{K}}
$$

поэтому в (4) можно положить

$$
C_{3}=b^{\varkappa}, \Lambda_{3}=b^{2 n \varkappa S} .
$$

Из теоремы 1 и соотношений (15), (17) и (18) получаем, что

$$
P\left(s_{0}\right) \ln \left(2+\frac{p_{s_{0}}}{\gamma_{5} n}\right) \leqslant p_{s_{0}}\left(\gamma_{6}+\varkappa \ln n\right)+\gamma_{7} \frac{n^{2}}{\ln ^{3} n} .
$$

Рассмотрим два случая.

1) Если $p_{s_{0}}<n^{2} / \ln ^{4} n$, то из (19) и неравенства $\ln \left(2+p_{s_{0}} /\left(\gamma_{5} n\right)\right)>\ln 2$ следует, что

$$
P\left(s_{0}\right) \leqslant \gamma_{8} \frac{n^{2}}{\ln ^{3} n}
$$

2) Если $p_{s_{0}} \geqslant n^{2} / \ln ^{4} n$, то при $n>n_{1}(\varepsilon)$ вьполняется неравенство

$$
\ln \left(2+\frac{p_{s_{0}}}{\gamma_{5} n}\right)>\left(1+\frac{\varepsilon}{2}\right)^{-1} \ln n
$$

и, как и в случае 1), из неравенства (19) вьводится оценка (14). Лемма доказана.

ДОКАЗАТЕЛЬСТВО ТЕОРЕМЫ ГЕЛЬФОНДА-ШНЕЙ ДЕРА. Допустим Противное: чИсла $a$ и $\alpha$ удовлетворяют условиям теоремы, но число $a^{\alpha}=e^{\alpha \ln a}$ алгебраическое. По лемме 2 построим функцию $F(z)$. При $\alpha \notin \mathbb{Q}$ все показатели $k+l \alpha$ в (11) различны, поэтому $F(z) \not \equiv 0$.

Пусть $t$ - наименьший порядок нуля функции $F(z)$ в точках

$$
s \ln a, \quad s=\overline{1, S}, \quad S=\varkappa+2,
$$

который достигается при $s=s_{0}$. Тогда из лемм 2 и 3 при $\varepsilon=1$ получаем, что

$$
\left[\frac{n^{2}}{\ln ^{2} n}\right]+(S-1) t \leqslant P\left(s_{0}\right) \leqslant(\varkappa+1) t+\gamma_{2} \frac{n^{2}}{\ln ^{3} n}
$$

иными словами,

$$
\left[\frac{n^{2}}{\ln ^{2} n}\right] \leqslant \gamma_{2} \frac{n^{2}}{\ln ^{3} n}
$$

что приводит к противоречию при достаточно больших $n$. Теорема доказана. 
ПРИмЕчАниЕ. Можно, но немного сложнее, получить противоречие и при $S=\varkappa+1$.

Укажем еще некоторые приложения доказанных теорем. Пусть $f_{1}(z), \ldots, f_{m}(z)$ - совокупность $E$-функций, т.е. функций вида (1), коэффициенты которых удовлетворяют условиям (2) и (4) с заменой условия (3) на более сильное требование:

$$
q_{n} a_{\nu} \in \mathbb{Z}_{\mathbb{K}}, \quad \nu=\overline{0, n}, \quad n=0,1, \ldots
$$

Далее, пусть функции $f_{1}(z), \ldots, f_{m}(z)$ составляют решение системы дифференциальных уравнений

$$
y_{i}^{\prime}=\sum_{j=1}^{m} Q_{i j}(z) y_{j}, \quad i=\overline{1, m}, \quad Q_{i j} \in \mathbb{K}(z) .
$$

Сначала сформулируем утверждение, близкое к лемме 2 [1, гл. 13].

ЛЕмма 4. Пусть совокупность Е-функиий $f_{1}(z), \ldots, f_{m}(z)$ составляет решение системы дифференциальных уравнений (20) и линейно независима над полем $\mathbb{C}(z)$. Тогда для любого достаточно большого натурального числа п существует последовательность линейных форм

$$
R_{k}(z)=\sum_{j=1}^{m} P_{k j}(z) f_{j}(z), \quad P_{k j}(z) \in \mathbb{K}(z), \quad k=1,2, \ldots
$$

такая, что определитель

$$
\Delta(z)=\operatorname{det}\left|P_{k j}(z)\right|_{k, j=\overline{1, m}}=\sum_{\gamma_{9} n<l<m n+\gamma_{10}} c_{l} \frac{z^{l}}{l !}
$$

отличен от нуля, причем

$$
c_{l} \in \mathbb{Z}_{\mathbb{K}}, \quad\left\lceil c_{l} \mid<e^{\gamma_{11} n}\right.
$$

ДокАЗАТЕЛЬСТво леммы почти дословно совпадает с соответствующими доказательствами из $[1$, гл. $13, \S 2]$ только с той разницей, что в равенстве (31) этой главы должен быть отброшен множитель $n !$.

Теорема 1 позволяет получить нетривиальную оценку порядка нуля определителя $\Delta(z)$ в точке $\alpha \neq 0$ и, тем самым, усилить результат леммы $3[1$, гл. 13$]$.

Полагая в теореме $1 f(z)=\Delta(z)$, получаем следующее

ПРЕДЛОЖЕНИЕ. Пусть выполнены все условия леммы 2 и $\alpha$-комплексное число, отличное от нуля и от полюсов коэффичиентов системы (20). Тогда существуют зависящие от $\alpha$ индексы $k_{1}, \ldots, k_{m}$ такие, что

$$
\operatorname{det}\left|P_{k_{i}, j}(\alpha)\right|_{i, j=\overline{1, m}} \neq 0
$$

$u$

$$
k_{1}+\cdots+k_{m}<\gamma_{12} \frac{n}{\ln n}
$$


С помощью обычных методов величину $k_{1}+\cdots+k_{m}$ удается оценить лишь тривиально величиной

$$
\operatorname{deg} \Delta(z)-\operatorname{ord}_{z=0} \Delta(z)+\gamma_{13} .
$$

Заметим, что для доказательства предложения не требуется, чтобы порядок нуля $\Delta(z)$ при $z=0$ был близок к $m n$.

В случае, когда функции $f_{1}(z), \ldots, f_{m}(z)$ являются $G$-функциями, в подобной лемме с помошью теоремы 2 при $|\alpha|<1$ аналогичным способом удается получить неравенство

$$
k_{1}+\cdots+k_{m}<\gamma_{14} n\left(\ln \frac{1}{|\alpha|}\right)^{-1} .
$$

В заключение заметим, что изложенный в настоящей статьеприем позволил В. В. Зудилину [4] дать полное обоснование предложенному Г. В. Чудновским [5] методу градуированных приближений Паде.

Московский государственный университет

Поступило

им. М.В. Ломоносова

17.05.96

E-mail: gal@nw.math.msu.su

\section{СПИСОК ЦИТИРОВАННОЙ ЛИТЕРАТУРЫ}

[1] Шидловский А. Б. Трансцендентные числа. М.: Наука, 1987.

[2] Siegel C.L. Über einige Anwendungen diophantischer Approximationen // Abh. Preuss. Wiss. Phys.-Math. Kl. 1929-1930. № 1. P. 1-70.

[3] Хейман У. Мероморфные функции. М.: Мир, 1966.

[4] Зудилин В.В. Об оценках снизу многочленов от значений некоторых целых функций // Матем. сб. 1996. Т. 187. №12. С. 57-86.

[5] Chudnovsky G. V. On some applications of diophantine approximations // Proc. Nat. Acad. Sci. USA. 1984. V. 81. March. P. 1926-1930. 